\title{
RISCO DE PERDA DE CONHECIMENTO NO CONTEXTO DA ADMINISTRAÇÃO PÚBLICA: UMA ANÁLISE BIBLIOMÉTRICA DA PRODUÇÃO CIENTÍFICA INDEXADA NA BASE DE DADOS SCIELO
}

\section{KNOWLEDGE LOSS RISK IN THE CONTEXT OF PUBLIC ADMINISTRATION: AN BIBLIOMETRIC ANALYSIS OF SCIENTIFIC PRODUCTION INDEXED IN THE SCIELO DATABASE}

\begin{abstract}
Leonardo Fernandes Souto
Programa de Pós-graduação em Políticas Públicas, Estratégia e Desenvolvimento da

Universidade Federal do Rio de Janeiro -PPED/UFRJ

lfsouto@gmail.com

Maria de Fátima Bruno-Faria

Programa de Pós-graduação em Políticas Públicas, Estratégia e Desenvolvimento da Universidade Federal do Rio de Janeiro - PPED/UFRJ e Faculdade de Administração e Ciências Contábeis da Universidade Federal do Rio de Janeiro - FACC/UFRJ fatimabruno@facc.ufrj.br
\end{abstract}

Submissão: $13 / 12 / 2020$

Aprovação: 02/12/2021

\section{RESUMO}

A partir da reforma administrativa e gerencial iniciada nos anos 1990, a administração pública passou a utilizar práticas de gestão até então próprias da iniciativa privada. Neste contexto, este estudo bibliométrico teve por objetivo analisar a representatividade do tema risco de perda de conhecimento, considerando sua interface com os temas gestão do conhecimento e gestão de riscos, no contexto da administração pública, a partir da produção científica indexada na base de dados SciELO. Os resultados demonstram a baixa representatividade dos temas risco de perda de conhecimento, gestão do conhecimento e gestão de riscos, sendo esta representatividade ainda menor quando relacionados com administração pública. Assim, percebe-se a importância da realização de futuros estudos sobre risco de perda de conhecimento, gestão do conhecimento e gestão de riscos, no contexto do setor público, de modo a contribuir para os avanços da produção científica relacionada a estes temas.

Palavras-chave: Risco de perda de conhecimento; Gestão do conhecimento; Gestão de riscos; Administração pública; Análise bibliométrica.

\begin{abstract}
From the administrative and managerial reform initiated in the 1990s, the public administration started to use management practices of the private initiative. In this context, this bibliometric study aimed to analyze the representativeness of the theme knowledge loss risk, considering your interface with the themes knowledge management and risk management, in the context of the public administration, from the scientific production
\end{abstract}


indexed in the database SciELO. The results demonstrate the absence of studies on knowledge loss risk and the low representativeness of the themes knowledge management and risk management, which is even lower when related to public administration. Thus, it is perceived the importance of carrying out future studies on knowledge loss risk, knowledge management and risk management, in the context of the public sector, in order to contribute to the advances in scientific production related to these themes.

Keywords: Knowledge loss risk; Knowledge management; Risk management; Public administration; Bibliometric analysis.

\section{Introdução}

De acordo com Ouverney (2005), o Brasil passou por três reformas administrativas: a primeira, durante as décadas de 1930 e 1940, relacionada à implantação do modelo burocrático weberiano na administração pública brasileira; a segunda, ocorrida nos anos 1960, "surgiu da necessidade de consolidar e aprofundar o processo de industrialização associado ao capital externo" (OUVERNEY, 2005, p. 4) sendo que "a instauração de novas agências da administração indireta - como autarquias, fundações e sociedades de economia mista - e a edição do Decreto-lei no 200/67 significaram a reformulação das estruturas administrativas" (OUVERNEY, 2005, p. 4), e a terceira, nos anos 1980 e 1990, foi a transição da administração burocrática para a gerencial a qual se referia à "aplicação de técnicas e ferramentas amplamente utilizadas na gestão empresarial em setores do Estado não incluídos no seu núcleo estratégico" (OUVERNEY, 2005, p. 4).

A reforma administrativa e gerencial pela qual passou a administração pública brasileira nos anos 1990 estava inserida em um movimento de abrangência internacional e "tanto no exterior quanto no Brasil, a estratégia consistiu inicialmente em valer-se de princípios e técnicas utilizados no mercado para redefinir os fundamentos da administração pública" (OUVERNEY, 2005, p. 5). Historicamente, os primeiros países a reconhecerem a necessidade de uma nova forma de administrar a organização do Estado e que realizaram uma reforma gerencial foram o Reino Unido, a Austrália e a Nova Zelândia, seguidos por outros, como o Brasil (BRESSER-PEREIRA, 2008).

Desde então, é possível notar a apropriação, pelo setor público, de diferentes práticas de gestão, comuns à iniciativa privada, almejando, sobretudo, uma maior eficiência da estrutura e dos processos administrativos e uma melhor qualidade dos serviços prestados. Campos e Baptista (2008), ao tratarem da reforma administrativa do Estado, reforçam a importância de se analisar a gestão do conhecimento na administração pública federal brasileira e consideram que a aplicação de práticas modernas de gestão aos processos de conhecimento pode proporcionar resultados e ações mais eficazes nas práticas gerenciais do setor público. É nesse cenário, de adoção de uma visão gerencial por parte do Estado, que se percebe a inclusão de diversas práticas e ferramentas de gestão no cotidiano da administração pública. Dentre estas práticas, é de interesse, neste estudo, a gestão do risco de perda de conhecimento, a qual mantém interface com a gestão do conhecimento e a gestão de riscos.

Nesse contexto, metodologicamente, esta pesquisa caracterizou-se como um estudo bibliométrico, a partir da análise de registros bibliográficos das publicações periódicas nacionais e internacionais indexadas na base de dados Scientific Electronic Library Online (SciELO). Trata-se de uma base de dados multidisciplinar que no momento da análise contava com 894.882 documentos indexados, publicados em 500 periódicos científicos, provenientes de 17 países: Brasil, Colômbia, México, Chile, Argentina, Espanha, África do 
Sul, Cuba, Portugal, Peru, Costa Rica, Uruguai, Bolívia, Paraguai, Equador, Venezuela e Índias Ocidentais, sendo que os projetos destes três últimos países se encontravam em desenvolvimento - o Brasil estava representado com 378 títulos de periódicos e 409.676 documentos indexados (SCIELO, 2020). A SciELO é o resultado de uma parceria entre a Fundação de Amparo à Pesquisa do Estado de São Paulo (FAPESP) e o Centro LatinoAmericano e do Caribe de Informação em Ciências da Saúde (BIREME), sendo que, desde 2002, conta com o apoio do Conselho Nacional de Desenvolvimento Científico e Tecnológico - CNPq (SCIELO, 2020).

Assim, este estudo teve por objetivo analisar a representatividade do tema risco de perda de conhecimento, considerando a interface entre gestão do conhecimento e a gestão de riscos, no contexto da administração pública, a partir da produção científica indexada na base de dados SciELO.

\section{Gestão do conhecimento, gestão de riscos e risco de perda de conhecimento}

Desde a década de 1970, há a ocorrência de estudos relacionando a gestão do conhecimento à administração pública, tendo como enfoque a vertente tecnológica, direcionada à aplicação de sistemas de informação (HENRY, 1974; GATES; 1975). Porém, os estudos de gestão do conhecimento começaram a se difundir a partir da obra de Nonaka e Takeuchi (1995) a qual enfatiza a diferenciação entre o conhecimento tácito e o explícito e propõe o Modelo SECI de conversão do conhecimento (socialização, externalização, combinação e internalização). Apesar de sua incorporação à iniciativa privada, com maior rapidez e escala, o setor público também vem se interessando pela gestão do conhecimento, seja na aplicação prática ou na reflexão teórica. Entretanto, para Razzaq et al. (2019, p. 940) "a gestão do conhecimento no setor público é uma área relativamente inexplorada de pesquisa e prática em especial com referência aos países em desenvolvimento e à Ásia”.

Massaro, Dumay e Garlatti (2015) realizaram uma revisão de literatura sobre gestão do conhecimento no setor público e de seus principais resultados destacam-se: poucos autores especializados no assunto; a maioria dos autores contribui apenas uma vez para o corpo de conhecimento; baixa cooperação internacional entre os autores; especialização de poucos periódicos no campo e a necessidade de ampliar a disseminação do tema; e concentração de estudos em alguns tópicos de pesquisa e em algumas áreas geográficas enquanto outros são subinvestigados.

Do ponto de vista da implementação prática, segundo Balasubramanian, Al-Ahbabi e Sreejith (2019, p. 5) "a natureza idiossincrática das instituições governamentais cria oportunidades peculiares e desafios para a implementação dos processos de GC”. Assim,

\footnotetext{
a introdução da gestão do conhecimento na administração pública significa implementar mudanças complexas, introduzir novos conhecimentos e, para isso, é necessário compreender este processo. Implementar e usar corretamente os princípios de gestão do conhecimento nos governos estaduais e locais pressupõe corretamente identificar os processos da organização, o desejo de educar os funcionários a longo prazo e identificar e implementar uma série de mudanças na organização do trabalho. (BUČKOVÁ, 2015, p. 395).
}

Nesse contexto, há exemplos recentes de estudos, sejam bibliométricos (SILVA; MIRANDA, 2018) teóricos (AL YAMI; AJMAL, 2019) ou relatos de aplicação prática (ADOBOR; KUDONOO; DANESHFAR, 2019; SPACEK; GATARIK, 2017), referentes à gestão do conhecimento no setor público.

Buscando compreender o contexto da gestão do risco de perda de conhecimento, da mesma forma que a gestão do conhecimento, a gestão de riscos é outra prática de gestão que vem sendo incorporada às rotinas da administração pública. Artigos referentes à gestão de 
riscos, em geral, podem ser encontrados desde a década de 1950 (ASZTÉLY, 1955; GALLAGHER, 1956), sendo, inicialmente, focados em riscos financeiros. Já documentos relacionando gestão de riscos à administração pública podem ser encontrados desde a década de 1980 (FIKSEL, 1981; KOEHLER, 1984).

De acordo com Smith e Toft (1998, p. 7), o setor público é tanto gerador de risco quanto regulador de risco e "existem poucas outras organizações que precisam gerenciar o amplo portfólio de riscos que o setor público enfrenta" sendo que para Hinna, Scarozza e Rotundi (2018, p. 110),

é necessário estudar o processo de implementação de práticas de gestão de riscos sobre formas de controle e prestação de contas em organizações do setor público, como forma de facilitar o alcance dos objetivos organizacionais e estratégicos, removendo ou mitigando os fatores que podem afetar o alcance das metas.

Nesse cenário, a gestão de riscos pode ser aplicada sob diferentes categorias de riscos, como por exemplo: risco financeiro, risco de crédito, risco operacional, risco de imagem, risco reputacional, risco ambiental, risco legal, risco de conhecimento, dentre outros. De interesse neste estudo, destaca-se a perspectiva do risco de conhecimento e, mais especificamente, do risco de perda de conhecimento, por ser uma abordagem que promove uma interface direta entre a gestão de riscos e a gestão do conhecimento, considerando-se, ainda, que o risco de perda de conhecimento relaciona-se com a proteção e retenção do conhecimento.

Diferentes autores, como por exemplo, Alhawari et al. (2012) e Durst e Henschel (2020), têm abordado a integração entre gestão do conhecimento e gestão de riscos. Quanto ao risco de perda de conhecimento, é possível citar, como exemplos, os estudos de IAEA (2006), Jennex (2009; 2014), Durst e Zieba (2017) e Massingham (2018). Massingham (2010) considera que a interseção entre as áreas de gestão do conhecimento e gestão de riscos tem como resultado a gestão do risco de conhecimento e apresenta um modelo conceitual para a gestão deste risco. Contudo, o risco de conhecimento pode ser desdobrado em diferentes categorias. Bratianuk (2018) identificou seis tipos de riscos de conhecimento: perda do conhecimento (knowledge loss), vazamento do conhecimento (knowledge leakage), transbordamento do conhecimento (knowledge spillover), terceirização do conhecimento (knowledge outsourcing), lacunas do conhecimento (knowledge gaps) e obsolescência do conhecimento (obsolete knowledge).

Nesse sentido, é importante destacar as definições de gestão do conhecimento, de gestão de riscos, de risco de conhecimento e de risco de perda de conhecimento, de modo a contextualizar, para o leitor, o escopo da pesquisa.

A gestão do conhecimento é entendida por Marr et al. (2003, p. 773) como "um grupo de processos e práticas usados por organizações para aumentar seu valor melhorando a eficácia da geração e aplicação de seu capital intelectual”. Segundo Vasile e Croitoru (2012, p. 253) a "gestão de riscos é o processo de identificação, análise e resposta aos riscos que a organização enfrenta e está exposta" sendo que "esse processo é contínuo e os resultados são dependentes das decisões assumidas quanto à aceitação, redução ou eliminação de riscos que afetam a consecução dos objetivos". Durst e Zieba (2019, p. 2) apresentam uma definição compreendendo o risco de conhecimento como "uma medida da probabilidade e gravidade dos efeitos adversos de qualquer atividade envolvida ou relacionada de alguma forma a conhecimentos que possam afetar o funcionamento de uma organização em qualquer nível”.

Para Jennex (2014, p. 93) o risco de perda de conhecimento é "o impacto causado na organização pela perda de uma fonte humana de conhecimento, geralmente um especialista, um trabalhador do conhecimento ou um gestor". Esta perda pode ser contextualizada, podendo o risco de perda de conhecimento ser tratado sob aspectos específicos. Daria (2018, p. 1740) analisa o risco de perda de conhecimento a partir da ótica da gestão de projetos e 
considera que "a experiência passada dos funcionários, adquirida em determinadas situações, pode ajudar a prever possíveis eventos e que, portanto, a tomada de decisões requer a extração e o armazenamento de conhecimentos que afetam a velocidade de identificação e resposta ao risco".

É nesse contexto que se apresentará, a seguir, o método; a análise da produção indexada, na base de dados SciELO, em relação aos temas de interesse deste estudo; a discussão e as considerações finais.

\section{Método}

Para a realização deste estudo bibliométrico, estabeleceu-se como universo da pesquisa o ambiente da base de dados SciELO. Segundo Teixeira, Iwamoto e Medeiros (2013, p. 425) "a bibliometria e campos afins têm como objetivo a mensuração do conhecimento ou a compreensão sobre sua produção". Ao tratarem da abordagem bibliométrica, os autores destacam que:

[...] não se trata apenas de identificar o que é mais produzido e por quem, mas usála de modo que nos permita identificar frestas que facultem perceber o que não está sendo produzido, abordagens não contempladas, buscar vozes não ouvidas e contrapô-las ao status quo. Com essa perspectiva talvez seja possível gerar conhecimento novo, sob enfoques novos [...] (TEIXEIRA; IWAMOTO; MEDEIROS, 2013, p. 445).

O levantamento de dados foi realizado por meio de diferentes buscas utilizando-se operadores booleanos. Primeiro, definiu-se o conjunto de temas e seus respectivos termos a serem pesquisados, considerando-se sinônimos ou termos relacionados, conforme Quadro 1 , a seguir.

Quadro 1 - Relação de temas e termos pesquisados

\begin{tabular}{|c|c|}
\hline $\begin{array}{l}\text { ONDE SE LE OS } \\
\text { TEMAS }\end{array}$ & FORAM PESQUISADOS OS TERMOS \\
\hline $\begin{array}{l}\text { Gestão do } \\
\text { conhecimento }\end{array}$ & $\begin{array}{l}\text { "gestão do conhecimento"; "gestão de conhecimento"; "gestión del } \\
\text { conocimiento"; "gestión de conocimiento"; "knowledge management" }\end{array}$ \\
\hline Gestão de riscos & $\begin{array}{l}\text { "gestão de riscos"; "gestão de risco"; "gestión de riesgos"; "gestión de } \\
\text { riesgo"; "risk management" }\end{array}$ \\
\hline $\begin{array}{l}\text { Administração } \\
\text { pública }\end{array}$ & 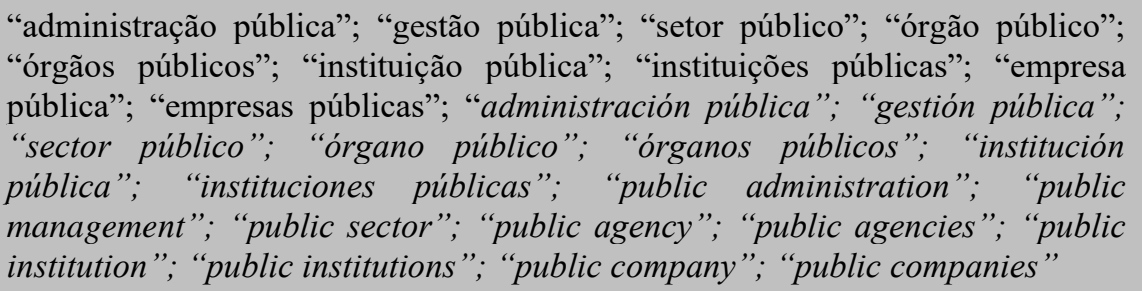 \\
\hline $\begin{array}{l}\text { Risco de perda de } \\
\text { conhecimento }\end{array}$ & $\begin{array}{l}\text { "risco de perda do conhecimento"; "risco de perda de conhecimento"; "riesgo } \\
\text { de pérdida del conocimiento"; "riesgo de pérdida de conocimiento"; } \\
\text { "knowledge loss risk" }\end{array}$ \\
\hline $\begin{array}{l}\text { Retenção do } \\
\text { conhecimento }\end{array}$ & $\begin{array}{l}\text { "retenção do conhecimento"; "retenção de conhecimento"; "retención del } \\
\text { conocimiento",; "retención de conocimiento"; "knowledge retention" }\end{array}$ \\
\hline $\begin{array}{c}\text { Perda do } \\
\text { conhecimento }\end{array}$ & $\begin{array}{l}\text { "perda do conhecimento"; "perda de conhecimento"; "pérdida de } \\
\text { conocimiento"; "pérdida del conocimiento"; "knowledge loss", }\end{array}$ \\
\hline $\begin{array}{l}\text { Proteção do } \\
\text { conhecimento }\end{array}$ & $\begin{array}{l}\text { "proteção do conhecimento"; "proteção de conhecimento"; "protección del } \\
\text { conocimiento"; "protección de conocimiento"; "knowledge protection" }\end{array}$ \\
\hline
\end{tabular}

Fonte: Elaboração própria. 
Para as buscas, realizadas em maio de 2020, utilizou-se o formulário de pesquisa avançada, fazendo-se uso da opção "Todos os índices". Quando da intenção de analisar a produção científica referente a um dos temas, utilizou-se o operador booleano OR, para pesquisar os termos sinônimos e relacionados, e, quando da intenção de cruzar diferentes temas, utilizou-se o operador booleano AND. Assim, as buscas com o operador OR permitiram identificar a quantidade de documentos que tratavam do tema pesquisado e as buscas com AND permitiram identificar a quantidade daqueles que relacionavam os temas cruzados (ver Quadro 2).

Nesse contexto, optou-se por considerar a revocação dos documentos recuperados. Rubi (2009, p. 85) esclarece que a revocação "pode ser mensurada por meio da relação entre o número de documentos relevantes sobre determinado tema, recuperados pelo sistema de busca, e o número total de documentos sobre o tema, existentes nos registros do mesmo sistema" enquanto que a precisão "pode ser mensurada por meio da relação entre os documentos relevantes recuperados e número total de documentos recuperados". Já para Lancaster (2004, p. 4) a revocação consiste na "capacidade de recuperar documentos úteis" enquanto que a precisão se refere à "capacidade de evitar documentos inúteis".

Quadro 2 - Relação de temas cruzados

\begin{tabular}{|c|}
\hline Gestão de riscos X Gestão do conhecimento \\
\hline Administração pública X Gestão de riscos \\
\hline Administração pública X Gestão do conhecimento \\
\hline Gestão do conhecimento X Retenção do conhecimento \\
\hline Gestão do conhecimento X Perda do conhecimento \\
\hline Gestão do conhecimento X Proteção do conhecimento \\
\hline Gestão de riscos X Retenção do conhecimento \\
\hline Gestão de riscos X Perda do conhecimento \\
\hline Gestão de riscos X Proteção do conhecimento \\
\hline Administração pública X Retenção do conhecimento \\
\hline Administração pública X Perda do conhecimento \\
\hline Admistração pública X Proteção do conhecimento \\
\hline
\end{tabular}

Fonte: Elaboração própria.

Para a análise, adotou-se a estatística descritiva, a partir do cálculo da frequência (quantidade) de ocorrências de publicações que tratavam de determinado tema e daquelas que relacionavam os cruzamentos realizados. Por fim, a partir dos resultados obtidos das buscas, fez-se uso dos seguintes filtros, da própria base de dados, para a realização de análises complementares: Ano de publicação; Periódicos; e WoS Áreas Temáticas, sendo, este último, referente a uma classificação temática disponível na própria base de dados, de acordo com as categorias da Web of Science (WoS). 


\section{Descrição e análise dos resultados}

\subsection{Análise dos temas pesquisados}

Em maio de 2020, a base de dados SciELO possuía 894.882 documentos indexados, no momento das buscas. Destes, 1.036 tratavam de gestão do conhecimento. Em relação aos estudos sobre gestão do conhecimento, na base de dados SciELO, estes começaram a ser publicados em 1999. Percebe-se uma evolução crescente nos anos seguintes, com pequenas oscilações a partir de 2010, sendo que o pico de publicação ocorreu em 2015, conforme Gráfico 1.

Gráfico 1 - Quantidade de documentos por ano, na base de dados SciELO, que tratavam de gestão do conhecimento

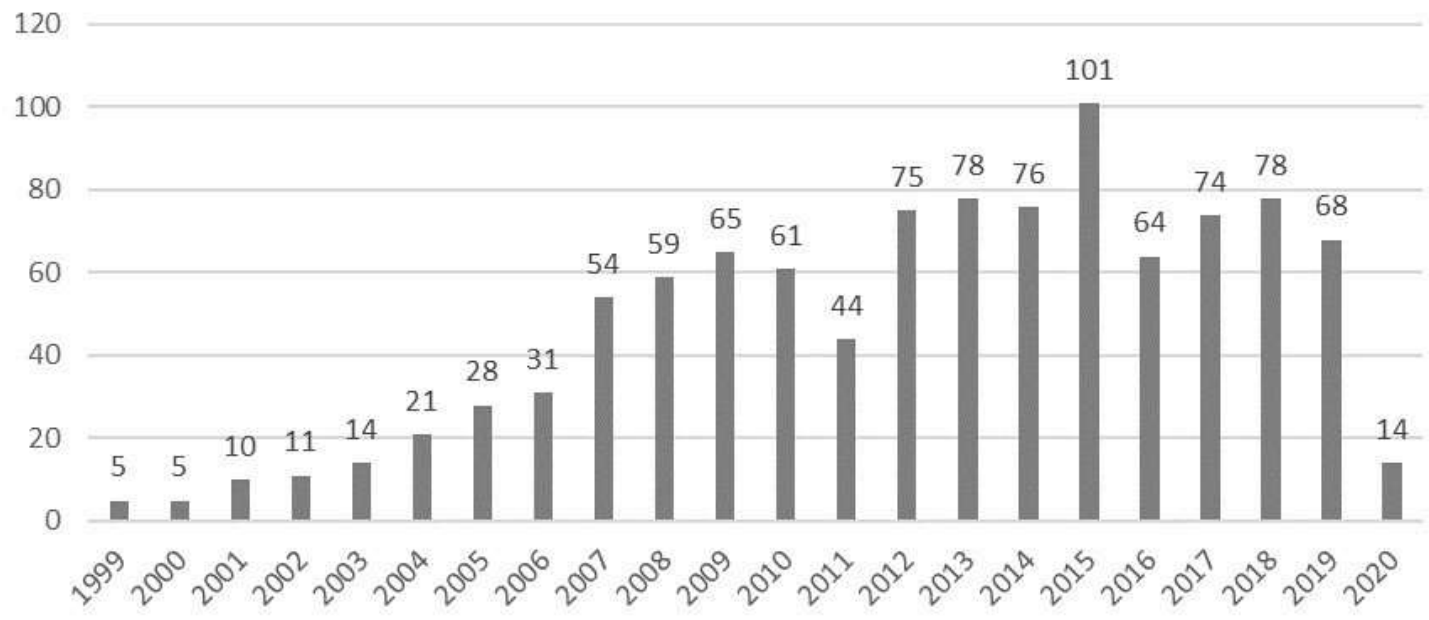

Fonte: Elaboração própria.

Dos documentos que tratavam do tema gestão do conhecimento, segundo a classificação "WoS Áreas Temáticas", apenas dez estavam classificados como Administração pública, tendo sido publicados nos periódicos: South African Journal of Economic and Management Sciences; Gestión y política pública; e Revista de Administração Pública. O primeiro estudo de gestão do conhecimento, classificado na Área de Administração pública, segundo a classificação "WoS Áreas Temáticas", foi publicado em 2005.

Já no que se refere aos estudos sobre gestão de riscos, dos 894.882 documentos indexados na base SciELO, 972 tratavam do tema. Estes estudos começaram a ser publicados em 1993. Percebe-se uma tendência de crescimento, cujo pico ocorreu no período de 2017 a 2019, conforme Gráfico 2.

Gráfico 2 - Quantidade de documentos por ano, na base de dados SciELO, que tratavam do tema gestão de riscos 


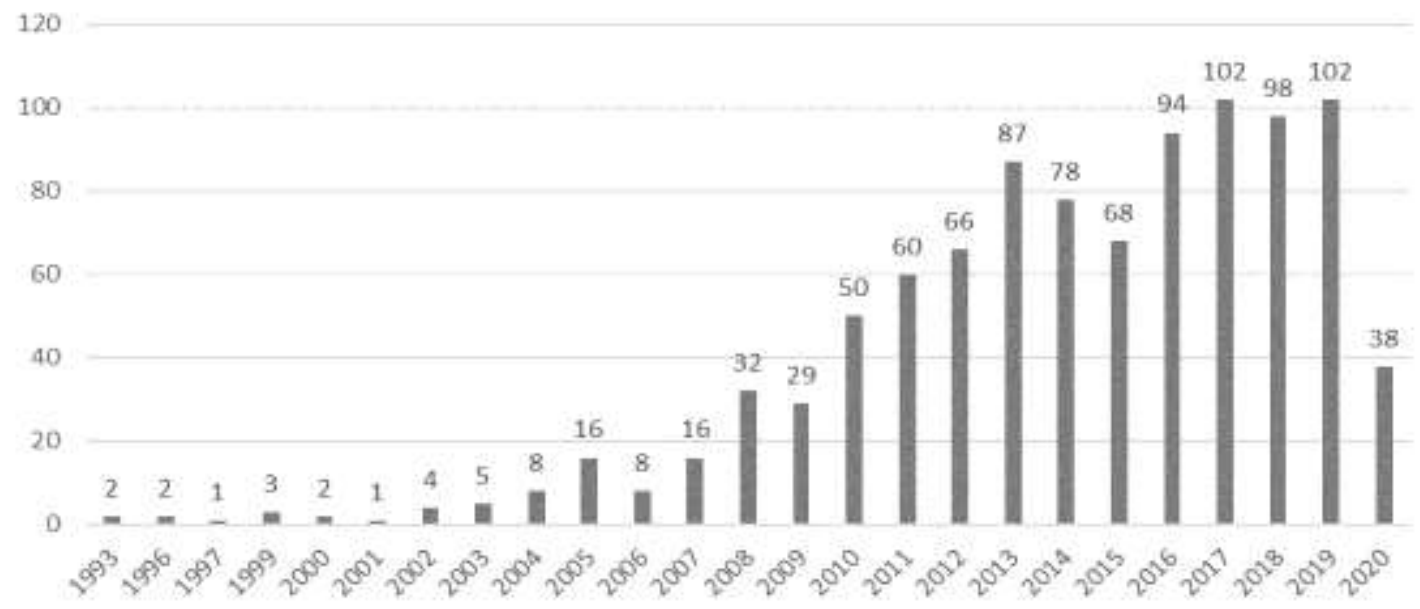

Fonte: Elaboração própria.

Dos documentos que tratavam do tema gestão de riscos, segundo a classificação "WoS Áreas Temáticas", 16 eram classificados como Administração pública, segundo a classificação "WoS Áreas Temáticas", tendo sido publicados nos periódicos South African Journal of Economic and Management Sciences; Gestión y política pública; e Revista de Administração Pública.

Quanto à representatividade do tema administração pública, dos 894.882 documentos indexados na base, 6.309 tratavam do assunto, tendo sido publicados a partir de 1968 . Destes, 1.185 estavam classificados como Administração pública, segundo a classificação "WoS Áreas Temáticas", sendo que, conforme Gráfico 3, o primeiro estudo foi publicado em 2005.

Gráfico 3 - Quantidade de documentos por ano, na base de dados SciELO, resultantes da busca administração pública e classificados como Administração pública segundo a classificação "WoS Áreas Temáticas"

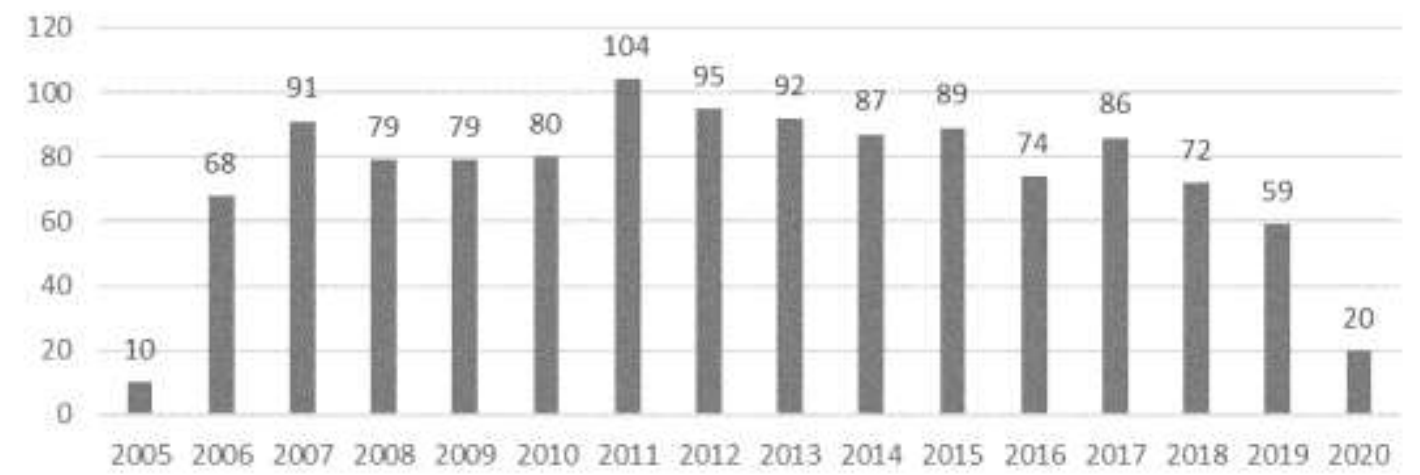

Fonte: Elaboração própria.

Dos 894.882 documentos indexados na base de dados, nenhum documento tratava do tema risco de perda de conhecimento. Porém, 32 estudos tratavam de retenção do conhecimento, 31 de perda de conhecimento e dez de proteção do conhecimento, conforme Tabela 1.

Tabela 1 - Quantidade de documentos, na base de dados SciELO, que tratavam de retenção do conhecimento, perda do conhecimento e proteção do conhecimento 


\begin{tabular}{lc}
\hline \multicolumn{1}{c}{ Tema } & Quantidade \\
\hline Retenção do conhecimento & 32 \\
Perda do conhecimento & 31 \\
Proteção do conhecimento & 10 \\
\hline
\end{tabular}

Fonte: Elaboração própria.

Apenas em relação ao tema perda de conhecimento foi encontrado um documento classificado como Administração pública, segundo a classificação "WoS Áreas Temáticas", sendo este publicado na Revista de Administração Pública.

\subsection{Análise dos cruzamentos dos temas pesquisados}

Dos 972 documentos que tratavam de gestão de riscos, ao realizar o cruzamento com gestão do conhecimento, foram encontrados seis estudos que relacionavam estes temas, sendo estes publicados nos periódicos Revista de Salud Pública; Journal of Technology Management \& Innovation; Revista EAN; Revista Facultad de Ciencias Económicas: Investigación y Reflexión; e Revista de la Asociación Española de Neuropsiquiatría.

Dos 6.309 documentos que tratavam do tema administração pública, ao realizar o cruzamento com gestão de riscos, foram encontrados 20 estudos que relacionavam estes temas, tendo sido publicados em 17 diferentes periódicos, do quais destacam-se a Revista de Administração Pública, com três artigos, e a Revista Derecho del Estado, com duas publicações. Os demais periódicos publicaram apenas um documento.

Dos 6.309 documentos que tratavam do tema administração pública, ao se fazer o cruzamento com gestão do conhecimento, foram encontrados 37 estudos que relacionavam os temas. Destes, seis eram classificados como Administração pública, segundo a classificação "WoS Áreas Temáticas".

Esses 37 documentos, resultantes do cruzamento dos temas administração pública e gestão do conhecimento, foram publicados em 23 periódicos, sendo que o periódico que mais teve publicações relacionadas foi a Revista de Administração Pública com seis estudos. Estes seis correspondem aos mesmos resultantes do cruzamento entre administração pública e gestão do conhecimento e classificados como Administração pública, segundo a classificação "WoS Áreas Temáticas".

Gráfico 4 - Quantidade de documentos na base de dados SciELO, por periódico, resultante do cruzamento dos temas administração pública e gestão do conhecimento 


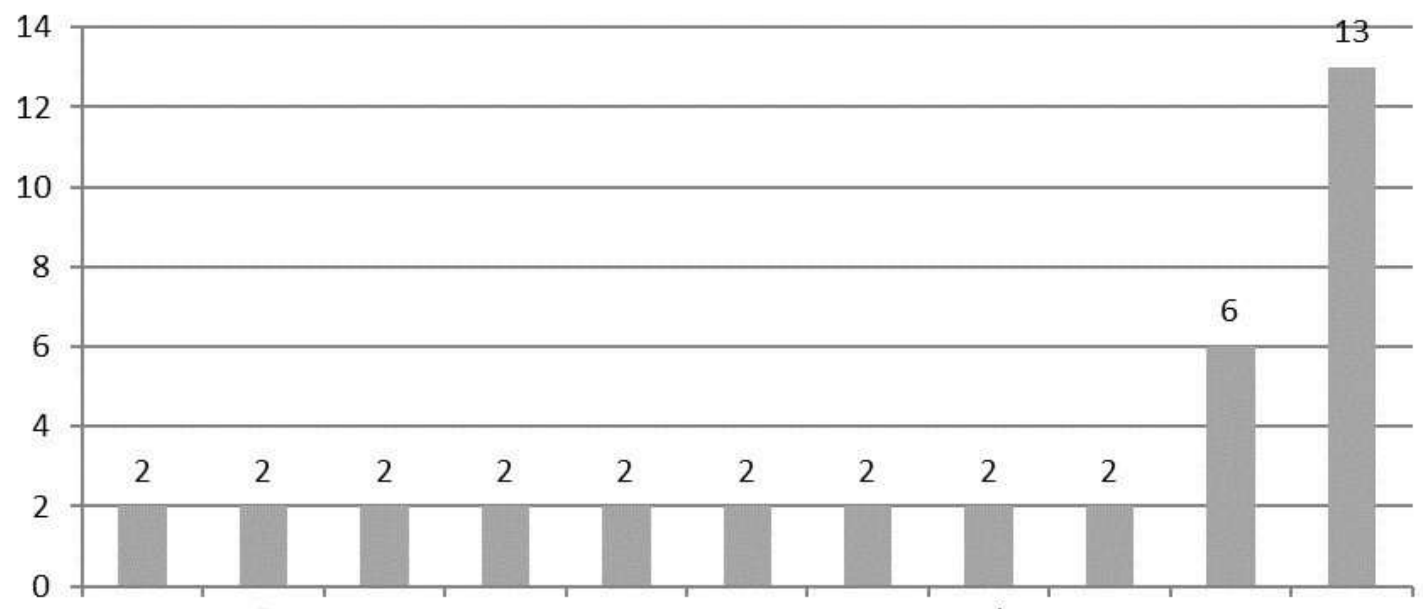

\begin{tabular}{|c|c|c|c|c|c|c|c|c|}
\hline 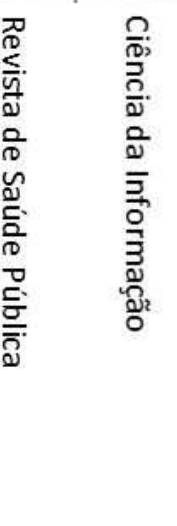 & 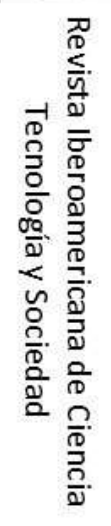 & 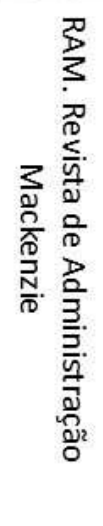 & 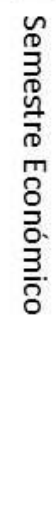 & 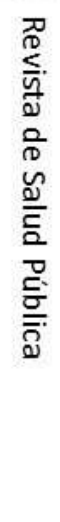 & 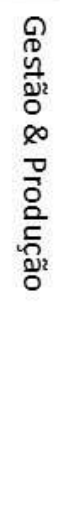 & 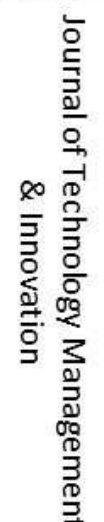 & 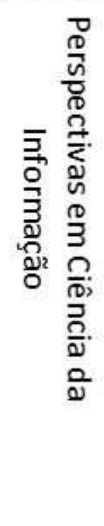 & 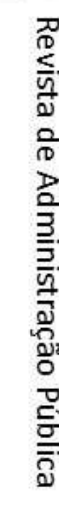 \\
\hline
\end{tabular}

Fonte: Elaboração própria.

Ao cruzar, individualmente, o tema gestão do conhecimento com os temas retenção do conhecimento, perda do conhecimento e proteção do conhecimento, foram encontrados quatro documentos que relacionavam gestão do conhecimento e retenção do conhecimento, dois que relacionavam gestão do conhecimento e perda de conhecimento e dois que relacionavam gestão do conhecimento e proteção do conhecimento, conforme Tabela 2.

Tabela 2 - Quantidade de documentos, na base de dados SciELO, relacionados ao cruzamento de gestão do conhecimento com retenção do conhecimento, perda do conhecimento e proteção do conhecimento

\begin{tabular}{lc}
\hline \multicolumn{1}{c}{ Cruzamentos de temas } & Quantidade \\
\hline Gestão do conhecimento X retenção do conhecimento & 4 \\
Gestão do conhecimento X perda de conhecimento & 2 \\
Gestão do conhecimento X proteção do conhecimento & 2 \\
\hline
\end{tabular}

Fonte: Elaboração própria.

Os estudos relacionados ao cruzamento de gestão do conhecimento com retenção do conhecimento, perda do conhecimento e proteção do conhecimento foram publicados em quatro periódicos: BAR - Brazilian Administration Review; Perspectivas em Ciência da Informação; Production; e Revista de Administração Pública. Dos documentos relacionados ao cruzamento de gestão do conhecimento com retenção do conhecimento, perda do conhecimento e proteção do conhecimento, apenas em relação ao cruzamento de gestão do conhecimento e perda de conhecimento é que foi encontrado um documento classificado como Administração pública, segundo a classificação "WoS Áreas Temáticas", sendo este publicado na Revista de Administração Pública. Apesar da interface entre gestão do conhecimento e gestão de riscos, não foi identificado nenhum documento resultante do 
cruzamento do tema gestão de riscos com os temas retenção do conhecimento, perda do conhecimento ou proteção do conhecimento.

Por fim, em relação ao cruzamento do tema administração pública com os temas retenção do conhecimento, perda do conhecimento ou proteção do conhecimento, apenas no que se refere ao tema perda de conhecimento foi identificado um documento, sendo, este, publicado na Revista de Administração Pública. Este documento é o mesmo resultante da busca perda de conhecimento e classificado como Administração Pública, segundo a classificação "WoS Áreas Temáticas", assim como resultante do cruzamento de gestão do conhecimento com perda de conhecimento e classificado como Administração pública, segundo a classificação "WoS Áreas Temáticas".

\subsection{Análise dos artigos sobre perda de conhecimento}

Não tendo sido recuperadas publicações na base de dados SciELO sobre o tema risco de perda de conhecimento, optou-se por realizar a análise daquelas relacionadas ao tema perda de conhecimento. Desta forma, em dezembro de 2020, realizou-se uma pesquisa complementar, que identificou a inserção de mais dois novos estudos sobre perda de conhecimento.

Ao analisar os documentos, identificou-se três concepções de perda de conhecimento: perda da consciência, perda de conhecimento tradicional e perda de conhecimento organizacional. Assim, apresenta-se, a seguir, a análise dos artigos relacionados à perda de conhecimento organizacional, por se tratar do contexto de interesse deste estudo.

Quadro 3 - Análise descritiva dos artigos sobre o tema perda de conhecimento organizacional, indexados na base de dados SciELO, em dezembro de 2020

\begin{tabular}{|c|l|l|}
\hline TíTULO & ANO & \multicolumn{1}{|c|}{ DESTAQUES } \\
\hline $\begin{array}{c}\text { Knowledge } \\
\text { forms in the } \\
\text { project } \\
\text { lifecycle: a } \\
\text { blueprint for } \\
\text { knowledge } \\
\text { management in } \\
\text { small creative } \\
\text { agencies in } \\
\text { Johannesburg }\end{array}$ & 2020 & $\begin{array}{l}\text { A pesquisa de Labuscagne (2020) utilizou o ciclo de vida do projeto, em } \\
\text { agências de criação - entendidas como organizações intensivas em } \\
\text { conhecimento e classificadas como pequenas e médias empresas - como um } \\
\text { dispositivo de organização para o delineamento de diferentes formas de } \\
\text { conhecimento em cada fase do projeto. O estudo contemplou oito } \\
\text { organizações em Joanesburgo, África do Sul, tendo sido realizadas oito } \\
\text { entrevistas com proprietários, gerentes ou diretores. Labuscagne (2020) } \\
\text { identificou quatro fatores para o risco de perda de conhecimento em pequenas } \\
\text { agências de criação: equipes pequenas dependem muito da tomada de decisão } \\
\text { espontânea; relutância em perder tempo para o registro, devido às altas } \\
\text { demandas do cliente do projeto; confiança excessiva na confiança dos } \\
\text { membros da equipe; e confiança excessiva na permanência da pessoa-chave. }\end{array}$ \\
\hline
\end{tabular}




\begin{tabular}{|c|c|c|}
\hline TÍTULO & ANO & DESTAQUES \\
\hline $\begin{array}{l}\text { A review of } \\
\text { knowledge } \\
\text { transfer tools } \\
\text { in knowledge- } \\
\text { intensive } \\
\text { organisations }\end{array}$ & 2020 & $\begin{array}{l}\text { O estudo Mazorodze e Buckley (2020) teve por objetivo identificar e revisar } \\
\text { as ferramentas de transferência de conhecimento usadas em organizações } \\
\text { intensivas em conhecimento e recomendar a melhor ferramenta de } \\
\text { transferência de conhecimento que pode ser usada nas organizações com o } \\
\text { propósito de aumentar a vantagem competitiva. Foi utilizada a estratégia de } \\
\text { Survey, com a aplicação de um questionário para a coleta de dados em } \\
\text { organizações intensivas em conhecimento na Namíbia, sendo que a amostra } \\
\text { contou com } 112 \text { participantes. O estudo conclui que a ferramenta mais eficaz } \\
\text { para transferência de conhecimento em organizações intensivas em } \\
\text { conhecimento são as comunidades de prática, seguida de mentoria, narrativa, } \\
\text { planos de sucessão, coaching e repositórios de conhecimento. Para } \\
\text { Mazorodze e Buckley (2020, p. 1) "as organizações intensivas em } \\
\text { conhecimento perdem propriedade intelectual quando funcionários } \\
\text { experientes se aposentam de seus empregos" e "para evitar a perda de } \\
\text { conhecimento, habilidades e experiência devem ser transferidas de } \\
\text { especialistas para não especialistas a tempo" sendo que "as ferramentas de } \\
\text { transferência de conhecimento permitem o compartilhamento de } \\
\text { conhecimento tácito entre os membros da equipe". Ao tratar do planejamento } \\
\text { da sucessão Mazorodze e Buckley (2020, p. 4) consideram que "quando os } \\
\text { funcionários se aposentam, as organizações enfrentam a perda de experiência } \\
\text { intelectual e institucional, memória e capital para a resolução de problemas" e } \\
\text { que a introdução dos planos de sucessão é uma forma para superar este } \\
\text { desafio e manter o conhecimento organizacional. }\end{array}$ \\
\hline $\begin{array}{l}\text { Knowledge } \\
\text { retention in a } \\
\text { platinum mine } \\
\text { in the North } \\
\text { West Province } \\
\text { of South Africa }\end{array}$ & 2018 & $\begin{array}{l}\text { Makhubela e Ngoepe (2018) estudaram a questão da retenção de } \\
\text { conhecimento em uma mina de platina na Província Noroeste da África do } \\
\text { Sul, por meio da realização de entrevistas semiestruturadas, com dez } \\
\text { empregados, dentre os quais, funcionários experientes e gerentes de } \\
\text { diferentes departamentos (RH, gestão de talentos, engenharia e TI) da } \\
\text { organização, sendo também utilizada a análise documental. Os autores } \\
\text { consideram que no combate à perda de conhecimento organizacional a } \\
\text { retenção de conhecimento desempenha um papel importante. Makhubela e } \\
\text { Ngoepe (2018) destacam que a perda de conhecimento nas organizações } \\
\text { ocorre quando os funcionários se aposentam mais cedo, encontram outro } \\
\text { emprego ou são atraídos por outras organizações. Makhubela e Ngoepe } \\
\text { (2018, p. 3) complementam que "a perda de funcionários como resultado de } \\
\text { demissões, dispensas, aposentadoria e realocação tem implicações de custo } \\
\text { consideráveis para a organização, pois leva à perda de conhecimento" e que } \\
\text { "o desafio atualmente enfrentado pelo RH é abordar a questão da perda de } \\
\text { conhecimento nas organizações". }\end{array}$ \\
\hline $\begin{array}{l}\text { Retenção de } \\
\text { conhecimento } \\
\text { em serviços }\end{array}$ & 2016 & $\begin{array}{l}\text { Gonzalez (2016) realizou um estudo com o objetivo de analisar um modelo } \\
\text { organizacional voltado para um prestador de serviço que promova a retenção } \\
\text { do conhecimento adquirido em seus diversos clientes. Foram realizadas } \\
\text { entrevistas com gestores de três sites full service, que prestam serviços a } \\
\text { empresas do segmento de bebidas, e também um gerente responsável pelo } \\
\text { Centro de Excelência da organização estudada. Para o autor a chance de } \\
\text { ocorrer a perda do conhecimento e de vantagem competitiva em empresas } \\
\text { prestadoras de serviço é grande quando elas não se estruturam com a } \\
\text { finalidade de resgatar o conhecimento diluído entre os diversos locais de } \\
\text { operação. Segundo Gonzalez (2016) o processo de retenção de conhecimento } \\
\text { nas empresas prestadoras de serviço deve ser estabelecido a partir de um } \\
\text { departamento específico que tenha como responsabilidades a identificação e o } \\
\text { resgate de melhores práticas e lições aprendidas entre os funcionários que } \\
\text { prestam serviços em diferentes clientes; e a promoção da integração desses } \\
\text { funcionários buscando disseminar o conhecimento tácito. No contexto do } \\
\text { estudo, este departamento é chamado de Centro de Excelência e tem como } \\
\text { principal função "garantir que o conhecimento adquirido e transformado nos } \\
\text { sites de prestação de serviço seja absorvido pela organização, ou seja, }\end{array}$ \\
\hline
\end{tabular}




\begin{tabular}{|c|c|c|}
\hline TÍTULO & ANO & DESTAQUES \\
\hline & & $\begin{array}{l}\text { preservar o conhecimento organizacional da empresa prestadora de serviço" } \\
\text { (GONZALEZ, 2016, p. 62). }\end{array}$ \\
\hline $\begin{array}{c}\text { Gestão do } \\
\text { conhecimento } \\
\text { numa } \\
\text { instituição } \\
\text { pública de } \\
\text { assistência } \\
\text { técnica e } \\
\text { extensão rural } \\
\text { do Nordeste do } \\
\text { Brasil }\end{array}$ & 2012 & $\begin{array}{l}\text { O estudo de Brito, Oliveira e Castro (2012) teve como tema a gestão do } \\
\text { conhecimento em uma instituição pública de assistência técnica e extensão } \\
\text { rural do Nordeste do Brasil e por objetivo a identificação da gestão do } \\
\text { conhecimento, a partir da percepção dos gestores da instituição, tendo feito } \\
\text { uso do modelo de Diagnóstico de Gestão do Conhecimento proposto por } \\
\text { Bukowitz e Williams (2002). A instituição investigada é um órgão que } \\
\text { executa políticas públicas de assistência técnica e extensão rural voltadas para } \\
\text { agricultores familiares. O estudo contou com a participação de } 90 \% \text { dos } 40 \\
\text { gestores envolvidos nos níveis tático e estratégico, em atividade durante os } \\
\text { meses de fevereiro a abril de 2011, que responderam ao questionário. Os } \\
\text { autores chamam a atenção para a contradição de que "uma organização que } \\
\text { dissemina saberes para agricultores familiares não possui internamente a } \\
\text { gestão formal do conhecimento que produz" e destacam ainda que apesar da } \\
\text { instituição, um órgão de educação não formal, ser criadora de conhecimento, } \\
\text { ele não é considerado seu negócio principal. Segundo Brito, Oliveira e Castro } \\
\text { (2012, p. 1341) "um dos problemas mais graves enfrentados pelas } \\
\text { organizações públicas é a perda de conhecimento em transferências, } \\
\text { rotatividade e aposentadorias de pessoal, pois tem como consequência a perda } \\
\text { de know-how e capital intelectual, já que muitas informações, conhecimentos } \\
\text { importantes e detalhes dos processos de trabalho ainda estão guardados } \\
\text { apenas na mente das pessoas". }\end{array}$ \\
\hline
\end{tabular}

Fonte: Elaboração própria.

Nesse contexto, chama a atenção o fato que das cinco publicações sobre perda de conhecimento, no contexto organizacional, quatro ocorreram nos últimos cinco anos e a mais antiga ainda não tem sequer dez anos.

Por fim, a análise temática, realizada a partir das palavras-chave dos cinco artigos apresentados no Quadro 3, permitiu a identificação de 19 assuntos:

Quadro 4 - Análise temática dos artigos sobre o tema perda de conhecimento organizacional, indexados na base de dados SciELO, em dezembro de 2020

\begin{tabular}{|l|l|}
\hline Agências criativas de Johannesburg & Mentoria \\
\hline Assistência técnica e extensão rural & Narrativa \\
\hline Ciclo de vida do projeto & Organizações intensivas em conhecimento \\
\hline Coaching & Planejamento de sucessão \\
\hline Comunidades de prática & Prestação de serviços \\
\hline Diagnóstico de gestão do conhecimento & Recuperação do conhecimento \\
\hline Distribuição do conhecimento & Repositórios de conhecimento \\
\hline Formas de conhecimento & Retenção do conhecimento \\
\hline Formas de gestão do conhecimento & Transferência de conhecimento \\
\hline Gestão do conhecimento & \\
\hline
\end{tabular}

Fonte: Elaboração própria.

\section{Discussão dos resultados}

Zanini, Pinto e Filippim (2012) realizaram uma análise bibliométrica aplicada à gestão do conhecimento, sendo o estudo feito a partir de citações referentes ao tema gestão do conhecimento, identificadas nos anais do Encontro Anual da Associação Nacional dos Programas de Pós-Graduação em Administração (EnANPAD), no período 2007 a 2010. Em suas conclusões, destacam que "a utilização de análise bibliométrica está se tornando cada 
vez mais necessária como indicadora da produção científica de determinada área e se converte em estratégia valiosa para a geração, sistematização e difusão do conhecimento".

A partir dos dados referentes à produção de gestão do conhecimento percebe-se a baixa representatividade do tema, assim como dos subtemas relacionados: risco de perda de conhecimento, retenção do conhecimento, perda do conhecimento e proteção do conhecimento. Apesar da análise inicial identificar que não houve incidência de documento indexado com o tema risco de perda de conhecimento, a pesquisa complementar, para o tema perda de conhecimento, realizada em dezembro de 2020, identificou o estudo de Labuscagne (2020) que aborda, tangencialmente, em seu conteúdo, a questão dos fatores para o risco de perda de conhecimento em pequenas agências de criação.

Nesse sentido, Zanini, Pinto e Filippim (2012) consideram que "a quantidade de produção científica de determinada área reflete o nível de formalidade que ela alcança. A Gestão do Conhecimento, por ser uma área nova possui um longo caminho a percorrer em relação ao seu desenvolvimento e adensamento científico".

Por sua vez, também é possível observar a baixa representatividade dos estudos de gestão do conhecimento no contexto da administração pública. Foram identificados apenas 37 documentos correspondentes a este cruzamento, além de que, segundo a classificação "WoS Áreas Temáticas", apenas dez sobre o tema gestão do conhecimento estavam classificados como Administração pública. Massaro, Dumay e Garlatti (2015), ao estudarem a gestão do conhecimento no contexto da administração pública destacam que há poucos autores especializados no campo e que o desenvolvimento de um corpo coeso da literatura sofre a interferência de vários obstáculos.

Rocha e Hoffmann (2014) realizam um estudo sobre a produção científica brasileira sobre gestão do conhecimento na base de dados Web of Science. Apesar de focado na produção científica brasileira, o estudo traz evidência do quanto é baixa a quantidade de pesquisas de gestão do conhecimento no setor público. Ao analisar o foco das áreas de pesquisa dos estudos, as autoras identificaram que aqueles relacionados à administração pública eram inferiores a 10.

Por fim, a Revista de Administração Pública destacou-se como o periódico com mais publicações relacionadas aos temas pesquisados. Sobretudo, em relação ao tema gestão do conhecimento, o estudo de Silva e Miranda (2018) confirma a contribuição deste periódico para a produção científica em gestão do conhecimento, ocupando a segunda posição. As autoras destacam ainda que ocorre uma concentração das produções em gestão do conhecimento em periódicos com melhor classificação Qualis, o que corresponde à realidade da Revista de Administração Pública, que possui Qualis A2.

\section{Considerações finais}

O objetivo deste estudo foi analisar a representatividade do tema risco de perda de conhecimento, considerando a interface entre gestão do conhecimento e a gestão de riscos, no contexto da administração pública. A identificação de apenas um documento sobre o tema risco de perda de conhecimento sinaliza que este é um assunto que se encontra em desenvolvimento e ainda está sendo consolidado. Os temas retenção do conhecimento, perda do conhecimento e proteção do conhecimento apresentaram baixa representatividade, de forma geral, assim como quando relacionados com os temas gestão do conhecimento e administração pública. Além disto, não foram identificados estudos que relacionassem os temas retenção do conhecimento, perda do conhecimento e proteção do conhecimento com gestão de riscos.

Percebeu-se que, de forma geral, os temas gestão do conhecimento, gestão de riscos 
e administração pública tinham baixa representatividade no escopo das publicações indexadas na base de dados SciELO, assim como os temas gestão do conhecimento e gestão de riscos tinham baixa representatividade dentre os temas que tinham sido objeto de atenção nas publicações sobre administração pública. Da mesma forma, constatou-se que, dentre os estudos que tratavam dos temas gestão do conhecimento, gestão de riscos e perda do conhecimento, havia baixa representatividade daqueles classificados como Administração pública, segundo a classificação "WoS Áreas Temáticas". Chamou a atenção o fato de que dos 6.309 documentos que atenderam ao critério de busca com o tema administração pública, apenas 1.185 eram classificados como Administração pública, segundo a classificação "WoS Áreas Temáticas". Tal fato sinaliza que se a busca tivesse sido realizada em campos específicos, como palavras-chave e título, o resultado poderia apresentar uma representatividade significativamente menor, uma vez que a busca foi realizada na opção de campos "Todos os índices", o que corresponde à busca de maior amplitude.

Os primeiros estudos de gestão do conhecimento indexados na SciELO datavam de 1999 e tiveram um aumento a partir de 2007, sendo que, destes estudos, o primeiro classificado como Administração pública, segundo a classificação "WoS Áreas Temáticas", foi publicado em 2005, coincidindo, este ano, com o início da publicação dos estudos indexados, na base de dados SciELO, referentes ao tema administração pública e classificados como Administração pública, segundo a "WoS Áreas Temáticas". Quanto à gestão de riscos, os primeiros estudos indexados na SciELO datavam de 1993 e tiveram um aumento a partir 2011.

Apesar da baixa frequência de estudos que relacionavam os temas gestão do conhecimento e gestão de riscos, com o tema administração pública, quando de sua ocorrência, destacou-se a Revista de Administração Pública como a principal fonte de disseminação.

Assim, as principais contribuições deste estudo referem-se à identificação da baixa representatividade da produção científica sobre os temas risco de perda de conhecimento, retenção do conhecimento, perda do conhecimento, proteção do conhecimento, gestão do conhecimento e gestão de riscos, no contexto da administração pública; à identificação da baixa diversificação de periódicos que cobrem tais temas; e à identificação e caracterização de estudos referentes ao tema perda de conhecimento.

Nesse contexto, percebe-se a oportunidade de contribuição para o desenvolvimento da área de administração pública, a partir da realização de estudos e relatos da aplicação de práticas referentes aos temas risco de perda de conhecimento, gestão do conhecimento e gestão de riscos, no setor público. Assim, a carência de pesquisas relacionadas ao risco de perda de conhecimento, demonstrada por este estudo, serve de estímulo para a aplicação prática de processos voltados para a gestão de risco de perda do conhecimento na Administração Pública, de modo a valorizar a gestão dos ativos de conhecimento. Tendo o setor público características muito específicas, sua realidade é muito distinta e isto e se constitui em um fator motivador para que as práticas de gestão do conhecimento e gestão de riscos sejam estudadas e repensadas.

Apresenta-se também a oportunidade para ampliação e diversificação da quantidade de periódicos da área de administração pública que publiquem estudos referentes aos temas risco de perda de conhecimento, retenção do conhecimento, perda do conhecimento, proteção do conhecimento, gestão do conhecimento e gestão de riscos, o que poderia ser feito, inclusive, mediante a publicação de fascículos temáticos/especiais, de modo a contribuir para que a área se desenvolva em relação aos referidos temas.

Por fim, apesar do fato de o estudo ter sido circunscrito aos documentos indexados pela base de dados SciELO; de ter se limitado à estatística descritiva, restringindo-se à 
frequência; de ter se baseado em revocação; e de estar sujeito aos parâmetros do sistema, no caso das informações analisadas a partir dos filtros disponibilizados pela respectiva base de dados; considera-se que os resultados contribuem para novos estudos sobre os temas risco de perda de conhecimento, gestão do conhecimento e gestão de riscos, no contexto do setor público, tendo em vista que se apresentam como um retrato da situação atual do cenário analisado e por servirem de referência para estudos futuros, comparativos, com outros temas e bases de dados de caráter internacional. 


\section{Referências}

ADOBOR, H.; KUDONOO, E; DANESHFAR, A. Knowledge management capability and organizational memory: a study of public sector agencies. International Journal of Public Sector Management, v. 32, n. 6, p. 671-687, 2019.

AL YAMI, M.; AJMAL, M. Pursuing sustainable development with knowledge management in public sector. VINE Journal of Information and Knowledge Management Systems, v. 49, n. 4, p. 568-593, 2019.

ALHAWARI, S. et al. Knowledge-based risk management framework for information technology project. International Journal of Information Management, v. 32, n. 1, p. 50-65, 2012.

ASZTÉLY, S. Det riskvilliga kapitalets förvaltning: en tänkbar metod att mäta dess effektivitet. Göteborg: Gumpert, 1955.

BALASUBRAMANIAN, S.; AL-AHBABI, S.; SREEJITH, S. Knowledge management processes and performance: the impact of ownership of public sector organizations. International Journal of Public Sector Management, v. 33, n. 1, p. 1-21, 2019.

BRATIANU, C. A holistic approach to knowledge risk. Management Dynamics in the Knowledge Economy, v. 6, n. 4, p. 593-607, 2018.

BRESSER-PEREIRA, L. C. O modelo estrutural de gerência pública. Revista de Administração Pública, v. 42, n. 2, p. 391-410, mar./abr. 2008.

BRITO, Lydia Maria Pinto; OLIVEIRA, Patrícia Webber Souza de; CASTRO, Ahiram Brunni Cartaxo de. Gestão do conhecimento numa instituição pública de assistência técnica e extensão rural do Nordeste do Brasil. Revista de Administração Pública, v. 46, n. 5, p. 1341-1366, set./out. 2012.

BUČKOVÁ, J. Knowledge management in public administration institutions. Procedia Economics and Finance, v. 34, p. 390-395, 2015.

BUKOWITZ, Wendi R.; WILLIAMS, Ruth L. Manual de gestão do conhecimento: ferramentas e técnicas que criam valor para a empresa. São Paulo: Bookman, 2002.

CAMPOS, M. M.; BAPTISTA, S. G. Gestão do conhecimento organizacional na administração pública federal em Brasília: um estudo exploratório. Revista Ibero-americana de Ciência da Informação (RICI), v. 1 n. 1, p. 181-197, jan./jun. 2008.

DARIA, S. Integrated knowledge loss risk management model in the project environment. In.: EUROPEAN CONFERENCE ON INDUSTRIAL ENGINEERING AND OPERATIONS MANAGEMENT, 2., 2018, Paris. Proceedings of the... Southfield: IEOM Society International, 2018.

DURST, S.; HENSCHEL, T. Knowledge Risk Management: state of research. In: DURST, S.; HENSCHEL, T. (Eds.). Knowledge Risk Management: from theory to praxis. Gewerbestrasse: Springer, 2020. p. 3-10. 
DURST, S.; ZIEBA, M. Knowledge risks - towards a taxonomy. International Journal of Business Environment, v. 9, n. 1, p. 51-63, 2017.

DURST, S.; ZIEBA, M. Mapping knowledge risks: towards a better understanding of knowledge management. Knowledge Management Research \& Practice, v. 17. n. 1, p. 1-13, 2019.

FIKSEL, J. Stress and stability: new concepts for risk management. Human Systems Management, v. 2, n. 1, p. 26-33, 1981.

GALLAGHER, R. B. Risk management: new phase of cost control. Harvard Business Review, v. 34, n. 5, p. 75-86, 1956.

GATES, B. Knowledge Management in the Technological Society: government by indicator. Public Administration Review, v. 35, n. 6, p. 589-593, 1975.

GONZALEZ, Rodrigo Valio Dominguez. Retenção de conhecimento em serviços. Perspectivas em Ciência da Informação, v. 21, n. 1, p. 42-65, mar. 2016.

HENRY, N. Knowledge Management: a new concern for public administration. Public Administration Review, v. 34, n. 3, p.189-196, 1974.

HINNA, A.; SCAROZZA, D.; ROTUNDI, F. Implementing Risk Management in the Italian Public Sector: hybridization between old and new practices. International Journal of Public Administration, v. 41, n. 2, p. 110-128, 2018.

INTERNATIONAL ATOMIC ENERGY AGENCY (IAEA). Risk management of knowledge loss in nuclear industry organizations. Viena: IAEA, 2006.

JENNEX, M. E. Assessing knowledge loss risk. In: Nickerson, R. C. \& Sharda, R. (Eds). Americas Conference on Information Systems, 15., 2009, San Francisco. Proceedings of the... Atlanta: Association for Information Systems, 2009.

JENNEX, M. E. A proposed method for assessing knowledge loss risk with departing personnel. VINE: The journal of information and knowledge management systems, v. 44, n. 2, p. 185-209, 2014.

KOEHLER, C. T. Public sector risk management: bibliography. Vance Bibliographies, Public Administration Series: Bibliography Issue, p. 1524, 1984.

LABUSCAGNE, Cobi W. Knowledge forms in the project lifecycle: a blueprint for knowledge management in small creative agencies in Johannesburg. South African Journal of Information Management, v. 22, n. 1, p. 1-9, 2020.

LANCASTER, F. W. Indexação e resumos: teoria e prática. 2.ed. Brasília: Briquet de Lemos, 2004.

MAKHUBELA, Salmon; NGOEPE, Mpho. Knowledge retention in a platinum mine in the North West Province of South Africa. South African Journal of Information 
Management, v. 20, n. 1, p. 1-8, 2018.

MARR, B. et al. Intellectual capital and KM effectiveness. Management Decision, v. 41, n.8, p. 771-781, 2003.

MASSARO, Maurizio; DUMAY, John C; GARLATTI, Andrea. Public sector knowledge management: A structured literature review. Journal of Knowledge Management, v. 19, n. 3, p. 530-558, May 2015.

MASSINGHAM, P. Knowledge risk management: a framework. Journal of Knowledge Management, v. 14, n. 3, p. 464-485, 2010.

MASSINGHAM, P. R. Measuring the impact of knowledge loss: a longitudinal study. Journal of Knowledge Management, v. 22, n. 4, p. 721-758, 2018.

MAZORODZE, Alfred H.; BUCKLEY, Sheryl. A review of knowledge transfer tools in knowledge-intensive organisations. South African Journal of Information Management, v. 22, n. 1, p. 1-6, 2020.

NONAKA, I.; TAKEUCHI, H. Criação de conhecimento na empresa: como as empresas japonesas geram a dinâmica da inovação. Rio de Janeiro: Campus, 1995.

OUVERNEY, A. M. Relações Estado, sociedade e mercado subjacentes aos padrões de organização estatal e o futuro da reforma administrativa. Cad. EBAPE.BR, v. 3, n. 2, p. 01-06, July 2005 .

RAZZAQ, S. et al. Knowledge management, organizational commitment and knowledgeworker performance: the neglected role of knowledge management in the public sector. Business Process Management Journal, v. 25, n. 5, p. 923-947, 2019.

ROCHA, M. B.; HOFFMANN, W. A. M. A produção científica brasileira sobre gestão do conhecimento na base de dados Web of Science. In: CONGRESSO BRASILEIRO DE GESTÃO DO CONHECIMENTO, 2014, Florianópolis. Anais...Florianópolis: SBGC, 2014.

RUBI, M. P. Os princípios da política de indexação na análise de assunto para catalogação: especificidade, exaustividade, revocação e precisão na perspectiva dos catalogadores e usuários. In: FUJITA, M. S. L. (Org.). A indexação de livros: a percepção de catalogadores e usuários de bibliotecas universitárias. Um estudo de observação do contexto sociocognitivo com protocolos verbais. São Paulo: Cultura Acadêmica, 2009. p. 81-93.

SCIELO. SciELO. São Paulo, 2020. Disponível em: < https://www.scielo.org>. Acesso em: 30 maio 2020.

SILVA, A. P. N.; MIRANDA, A. C. D. Gestão do conhecimento no setor público: um estudo sobre os artigos publicados em periódicos nacionais no período 2005-2015. Encontros Bibli: revista eletrônica de biblioteconomia e ciência da informação, Florianópolis, v. 23, n. 52, p. 73-83, maio 2018.

SMITH, D.; TOFT, B. Risk and Crisis Management in the Public Sector: Editorial: Issues in 
Public Sector Risk Management. Public Money \& Management, v. 18, n. 4, p. 7-10, Oct./Dez. 1998.

SPACEK, D.; GATARIK, E. Knowledge Management and Czech Self-Governments: empirical investigations into the application of knowledge management to public administration in the Czech Republic. The NISPAcee Journal of Public Administration and Policy, v. 10, n. 1, p. 201-220, jun. 2017.

TEIXEIRA, Maria Luisa Mendes; IWAMOTO, Helga Midori; MEDEIROS, Ana Lúcia. Estudos bibliométricos (?) em administração: discutindo a transposição de finalidade. Administração: Ensino e Pesquisa, v. 14, n. 3, p. 423-452, set. 2013.

VASILE, E.; CROITORU, I. Integrated Risk Management System: key factor of the management system of the organization. In: BANAITIENE, N. (Ed.) Risk Management: current issues and challenges. London: IntechOpen, 2012. p. 253-284.

ZANINI, G. B.; PINTO, M. D. S.; FILIPPIM, E. S. Análise bibliométrica aplicada à gestão do conhecimento. Conhecimento Interativo, v. 6, n. 2, p. 124-140, 2012. 